\title{
Iron Deficiency in Parkinsonism: Region-Specific Iron Dysregulation in Parkinson's Disease and Multiple System Atrophy
}

\author{
Naomi P. Visanji ${ }^{\mathrm{a}, *}$, Joanna F. Collingwood ${ }^{\mathrm{b}}$, Mary E. Finnegan ${ }^{\mathrm{b}}$, Anurag Tandon ${ }^{\mathrm{c}}$, Emily House ${ }^{\mathrm{d}}$ \\ and Lili-Naz Hazrati ${ }^{\mathrm{c}}$ \\ ${ }^{\mathrm{a}}$ Morton and Gloria Shulman Movement Disorders Centre, Toronto Western Hospital, Toronto, ON, Canada \\ ${ }^{\mathrm{b}}$ School of Engineering, University of Warwick, Coventry, UK \\ ${ }^{\mathrm{c}}$ Tanz Centre for Research in Neurodegenerative Disease, University of Toronto, ON, Canada \\ ${ }^{\mathrm{d}}$ The Birchall Centre, Keele University, Staffordshire, UK
}

\begin{abstract}
Alpha synuclein pathology is widespread and found in diverse cell types in multiple system atrophy (MSA) as compared to Parkinson's disease (PD). The reason for this differential distribution is unknown. Regional differences in the distribution of iron are associated with neurodegenerative diseases, and here we characterize the relationship between iron homeostasis proteins and regional concentration, distribution and form of iron in MSA and PD.

In PD substantia nigra, tissue iron and expression of the iron export protein ferroportin increased, while the iron storage protein ferritin expression was unchanged. In the basis pontis of MSA cases, increased total iron concentration coupled with a disproportionate increase in ferritin in dysmorphic microglia and a reduction in ferroportin expression. This is supported by isothermal remanent magnetisation evidence consistent with elevated concentrations of ferritin-bound iron in MSA basis pontis.

Conventional opinion holds that excess iron is involved in neurodegeneration. Our data support that this may be the case in PD. While region-specific changes in iron are evident in both PD and MSA, the mechanisms of iron dysregulation appear quite distinct, with a failure to export iron from the MSA basis pontis coupling with significant intracellular accumulation of ferritin iron. This pattern also occurs, to a lesser extent, in the MSA putamen. Despite the excess tissue iron, the manner of iron dysregulation in MSA is reminiscent of changes in anemia of chronic disease, and our preliminary data, coupled with the widespread pathology and involvement of multiple cell types, may evidence a deficit in bioavailabile iron.
\end{abstract}

Keywords: Parkinson's disease, multiple system atrophy, iron, ferritin, inflammation, ferroportin

\section{INTRODUCTION}

Multiple system atrophy (MSA) and Parkinson's disease (PD) are both neurodegenerative disorders

\footnotetext{
${ }^{*}$ Correspondence to: Naomi Visanji, Morton and Gloria Shulman Movement Disorders Centre, Toronto Western Hospital, 399 Bathurst Street, Toronto, ON, M5T 2S8, Canada. Tel.: +1 416 603 6482; Fax: +1 416603 5004; E-mail: naomi.visanji@ uhnresearch.ca.
}

characterised by aggregation of intracellular alpha synuclein. However, a key difference between these two synucleinopathies is the distribution of the underlying pathology. In PD alpha synuclein-positive aggregates, termed Lewy bodies, are restricted to neurons and found in a limited number of structures including the substantia nigra (reviewed in [1]). However, in MSA, multiple regions and multiple cell types are affected, with insoluble deposits of alpha synuclein 
found in both neurons and glia accompanied by widespread neurodegeneration apparent in many brain regions (reviewed in [1]).

Disrupted iron homeostasis has long been associated with neurodegenerative diseases, yet the role of iron in neurodegeneration remains elusive. Within the brain, iron is vital to many different cell types for many processes, including oxidative phosphorylation, myelin synthesis, nitric oxide metabolism, neurotransmitter synthesis and oxygen transport. However, excess iron has been shown to lead to the production of reactive oxygen species which in turn can lead to cellular damage and degeneration (reviewed in [2]). The oxidation state of iron in the tissue is therefore particularly important, and tightly regulated processes involving many proteins ensure that bioavailable highly reactive $\mathrm{Fe}$ (II) is minimised, with the majority being bound as $\mathrm{Fe}$ (III) in the course of normal brain iron homeostasis. Iron demonstrably affects, and is affected by, the aberrant peptide aggregation observed in neurodegenerative disease, including alpha synuclein, and recent evidence indicates that alpha synuclein is a cellular ferrireductase [3], and as recently demonstrated in an alpha synuclein knockout mouse model, may play a significant role in regulation of iron homeostasis [4]. Changes in iron at the cellular and sub-cellular scale are reflected in detectable disease-related shifts in the regional distribution of iron in the brain [5]. It has been shown that high iron levels correlate with regions of neurodegeneration, with evidence of increased iron in the PD and MSA substantia nigra and in the MSA striatum, particularly in putamen $[6,7]$. Within the brain, the majority of iron is sequestered within ferritin, a $450 \mathrm{kDa}$ protein found in every cell type, each protein capable of storing up to 4500 iron ions [8,9]. Ferritin levels are typically closely coupled to iron levels, such that increased iron leads to increased ferritin production. However, in PD substantia nigra there is evidence of a loss of this coupling such that iron is increased, but levels of ferritin are reportedly decreased $[6,7]$. This may lead to an increase in free, reactive iron, although this remains to be elucidated.

Recent advances in the understanding of iron homeostasis and in the application of modern techniques able to probe the status of iron within the brain allow for a more detailed investigation of this complex relationship. We studied the relationship between iron homeostasis proteins and the levels, distribution and form of iron in control, MSA and PD brain with a view to investigating a potential role of iron in the differing pathology of these two related neurodegenerative diseases.

\section{MATERIALS AND METHODS}

Tissue

All human brain tissue was obtained from the Canadian Brain Tissue Bank. Each brain was bisected at the time of death with one side dissected rapidly and different regions of the brain frozen at $-80^{\circ} \mathrm{C}$ and the other half immersed in $10 \%$ buffered formalin for subsequent blocking and embedding in paraffin. The median post mortem delay was 10 hours, with a range of 2-20 hours. Distributions of group size, sex, and mean age \pm SEM were: control $(n=3 ; 2$ male, 1 female; ages 47, 63 and 78 years); $\operatorname{PD}(n=3 ; 2$ male, 1 female; ages 56, 73 and 79 years); MSA ( $n=3 ; 2$ male, 1 female; ages 46, 66 and 76 years). For MSA the mean disease duration was $6 \pm 1.1$ years and all three cases presented clinically with MSA-P. The mean duration disease for PD cases was $21 \pm 3.8$ years and presented as akinetic-rigid. The diagnosis of all cases was confirmed neuropathologically. The brain areas used for western blotting, graphite furnace and Isothermal Remanent Magnetisation (IRM) analysis were from the frozen side whereas brain tissue used for immunohistochemistry was from the formalin fixed side of the brain. Different areas of interest included the substantia nigra (pars compacta), the basis pontis (pons) (both white and grey matter) and the lentiform nucleus (putamen, external/internal segments of the globus pallidus complex).

\section{Western blotting}

Frozen brain tissue was homogenised in 10x volume homogenisation buffer $(100 \mathrm{mM} \mathrm{NaCl}, 50 \mathrm{mM}$ Tris, $1 \mathrm{mM}$ EDTA, $1 \%$ CHAPS, broad spectrum protease inhibitor cocktail (Sigma-Aldrich, St. Louis, MO). After 20 minutes of incubation at $4{ }^{\circ} \mathrm{C}$, homogenates were centrifuged at $20,000 \times g$ for 5 minutes. Protein concentration was determined by BCA assay (Thermo Scientific, Waltham, MA). Western blotting was carried out according to standard protocols. Briefly, samples were heated for 10 minutes at $70^{\circ} \mathrm{C}$ in $25 \%$ NuPage ${ }^{\circledR}$ LDS sample buffer (Invitrogen, Carlsbad, CA). Proteins were then separated on 4-12\% Bis-Tris NuPage ${ }^{\circledR}$ Novex ${ }^{\circledR}$ Mini gels (Invitrogen, Carlsbad, CA) and transferred onto nitrocellulose membranes (Life Sciences, Frederick, CO). Blots were probed with antibodies to ferritin (SigmaAldrich), ferroportin (kind donation from Dr D Haile), transferrin receptor (GeneTex) and Glyceraldehyde 3-phosphate dehydrogenase (GAPDH, SigmaAldrich). Bound HRP-conjugated 
anti-mouse or anti-rabbit IgG (Sigma-Aldrich, St. Louis, MO) were revealed by chemiluminescence using ECL Plus (GE Healthcare, UK) and detected with a Storm 860 fluorescent imager. Protein levels were quantified by densitometric analysis using ImageQuant software (GE Healthcare, UK). All proteins were expressed as percent of GAPDH to control for variability in volume loaded. For each protein measured by western blot, 1 sample per patient was run on each gel for a total of 3 control samples, 3 PD samples and 3 MSA samples. Analysis was performed on a single blot and expressed as the mean \pm s.e.m of the 3 samples in a given group.

\section{Immunohistochemistry}

Formalin-fixed brain tissue was embedded in paraffin blocks, sectioned at $5 \mu \mathrm{m}$ and mounted on glass slides. Immunohistochemistry was performed according to standard protocols using antibodies for ferritin (SigmaAldrich), ferroportin (kind donation from Dr D Haile) and ionized calcium binding adaptor molecule 1 (iba1) (Abcam) and all tissue was counterstained with Mayer's Haematoxylin. Immunostained sections were scanned at 40x using a Scanscope digital slide scanner (Aperio Technologies, Inc., Vista, CA). Image files were viewed with ImageScope (Aperio Technologies, Inc., Vista, CA). Positive pixel analysis of iba1 stained tissue was carried out using a custom designed algorithm in ImageScope. The mean number of iba1 positive pixels in 3 equal sized regions of each sample was calculated.

\section{Superconducting quantum interference device (SQuID) magnetometry}

The fraction of brain iron that is contained in the iron storage protein ferritin (the ferritin core) is present as nanoscale iron oxide clusters, which typically have a ferrihydrite-like mineral structure. The magnetic properties of these ferritin cores are well-documented, and so magnetic analysis of tissue can be used to quantify the signal arising from the ferritin cores. If total iron concentration is measured subsequent to the magnetic analysis, the proportion of iron bound in ferritin may be estimated. In practice, a variety of iron biomineral phases are observed in ferritin cores, and the extent to which ferritin is clustered within tissue may vary; both factors can potentially affect the magnetic properties of the tissue. It should also be noted that other forms of bio-iron may contribute to IRM signal at 5 Kelvin (K), including neuromelanin-bound iron [10] and iron oxide deposits including magnetite which may be associated with pathologic features such as amyloid plaques $[11,12]$.

The parameter used to identify the signal from ferritin cores in the tissue was Isothermal Remanent Magnetisation (IRM). In this measurement, samples are exposed to a magnetic field. Biological material is primarily diamagnetic, which results in a negative linear magnetic response. This gives the 'background' signal, onto which the small but detectable positive response from the ferritin cores is superimposed. At body temperature, the positive response from the ferritin cores vanishes as soon as the applied magnetic field is removed. However, at very low temperatures (below approximately $20 \mathrm{~K}$ ), the ferritin cores retain, for a temporary period, a magnetisation that can be detected and quantified using the sensitivity of a SQuID magnetometer. The signal from normal ferritin cores is almost absent at higher temperatures, making it possible to estimate their concentration in a known mass of tissue. This approach, to analyse biomineralized iron in human tissue, is now well established and described elsewhere [13].

Fresh-frozen samples were dissected to obtain the region of interest, and then prepared for SQuID measurement by freeze drying and compressing each sample into a dense pellet. Acid-washed ceramic blades and non-metallic piston rods were used for cutting and compression respectively, to avoid environmental contamination of the samples with iron-rich particulates.

A Quantum Design MPMS-5S SQuID was used to measure the isothermal remanent magnetisation (IRM) at $150 \mathrm{~K}, 50 \mathrm{~K}$ and $5 \mathrm{~K}$. A demagnetising sequence was run prior to each IRM measurement to ensure that the initial condition for each sample was the same. IRM was obtained by applying a magnetic field of 1.0 Tesla, measuring the magnetic moment (the response from the tissue), and then removing the magnetic field. The IRM signal decays after the magnetic field is removed, so the remanent magnetisation is sampled eight times over approximately 10 minutes, and the final four readings (where the decay curve has stabilised) are averaged to obtain a measurement of the IRM; the error is the standard deviation for these four values. Only material capable of retaining a magnetic moment after application and removal of the 1.0 Tesla field can contribute to the IRM signal; this excludes the significant majority of biological material. The ferrihydrite-like iron oxide cores normally found in ferritin are assumed to be the primary contributor to the IRM signal at $5 \mathrm{~K}$, 
as tested by measurement of IRM saturation curves, and evidenced by loss of IRM signal at higher temperatures. All values were corrected for dry sample mass. Correction for finite sample length was applied using the method outlined by Hautot et al. [13].

\section{Graphite furnace atomic absorption spectroscopy}

Samples were digested using a Mars Xpress microwave (CEM Microwave Technology Ltd, UK) and analysed with an AAnalyst 600 atomic absorption spectrometer with a transversely heated graphite atomizer and longitudinal Zeeman-effect background corrector (Perkin Elmer, UK). The microwave digestion temperature program, GFAAS instrument parameters and calibration procedure were as described previously [14].

Freeze-dried tissue samples from the SQuID magnetometry analysis were dried to a constant weight (36-231 mg dry wt) in Teflon ${ }^{\odot}$ vessels and microwave digested in $1 \mathrm{~mL}$ nitric acid $\left(\mathrm{HNO}_{3}\right.$ analytical reagent grade $15.8 \mathrm{M}$, Fischer Scientific, UK) and $1 \mathrm{~mL}$ hydrogen peroxide $\left(\mathrm{H}_{2} \mathrm{O}_{2}\right.$, ARISTAR grade $\left.30 \% \mathrm{w} / \mathrm{v}\right)$. After cooling, $3 \mathrm{~mL}$ ultrapure water (conductivity $\leq 6.8 \times 10^{-6} \mathrm{~S} \mathrm{~m}^{-1}$ ) was added to each vessel, digest volumes were determined gravimetrically and ranged from 4.44 to $4.82 \mathrm{~mL}$. Digests were yellow and clear with no precipitate or fatty residue visible. Iron concentration was determined in 800 and 200 fold dilutions of the digests $(12.5 \mu \mathrm{L}$ and $50 \mu \mathrm{L}$ of digest diluted to $10 \mathrm{~mL}$ in $1 \% \mathrm{HNO}_{3}$, respectively). Vessels were cleaned by digesting with $1 \mathrm{~mL} \mathrm{HNO}_{3}$, rinsing with ultrapure water and storing in $5 \% \mathrm{HCl}$.

Samples were analysed in two batches. In each batch quality assurance (QA) samples included three method blanks, one reagent spike and two standard reference materials (NIST SRM 1566B oyster tissue and IAEA-407 fish homogenate). Two additional vessels containing spiked standard reference materials were included in the first batch. These QA were dried, digested and diluted, including the further 800 fold dilution, as for the tissue digests.

The mean of iron concentrations measured in 800 fold dilutions of the $\mathrm{ca} 5 \mathrm{~mL}$ method blank digests in batch one was $0.15 \mu \mathrm{gL}^{-1}$ (S.D. $0.34 \mu \mathrm{gL}^{-1}, n=3$ ) and $0.17 \mu \mathrm{g} \mathrm{L}^{-1}$ (S.D. $0.05 \mu \mathrm{g} \mathrm{L}^{-1}, n=3$ ) in batch two. Background contamination was corrected for by subtraction of the mean plus three standard deviations of the method blanks analysed in the same batch. This corresponded to $31.2 \mu \mathrm{g} \mathrm{g}^{-1}$ dry wt (S.D. $10.2 \mu \mathrm{g} \mathrm{g}^{-1}$, $n=9$ samples) in batch one and $23.3 \mu \mathrm{gg}^{-1}$ dry wt (S.D. $7.1 \mu \mathrm{g} \mathrm{g}^{-1}, n=13$ samples) in batch two.
The reagent spike recoveries were $97.2 \%$ and $103.5 \%$. The measured IAEA407 iron concentrations were $166.4 \mu \mathrm{gg}^{-1}$ dry wt (S.D. $0.9 \mu \mathrm{gg}^{-1}$ dry wt), and $147.2 \mu \mathrm{gg}^{-1}$ dry wt (S.D. $1.0 \mu \mathrm{gg}^{-1}$ dry wt), compared to a certified value of $146 \mu \mathrm{g} \mathrm{g}^{-1}$ (95\% CI $\left.3 \mu \mathrm{g} \mathrm{g}^{-1}\right)$. The measured SRM1566B iron concentrations were $205.0 \mu \mathrm{gg}^{-1}$ dry wt (S.D. $2.3 \mu \mathrm{gg}^{-1}$ ) and $173.6 \mu \mathrm{g} \mathrm{g}^{-1}$ dry wt (S.D. $0.9 \mu \mathrm{g} \mathrm{g}^{-1}$ ) compared to the certified value of $205.8 \pm 6.8 \mu \mathrm{gg}^{-1}$. Spike recoveries from SRM1566B and IAEA407 were $103.7 \%$ and $92.5 \%$ respectively.

The calibration curves had a correlation coefficient $\geq 0.998$, and instrumental detection limits of $0.14 \mu \mathrm{g} \mathrm{L}^{-1}$ and $0.21 \mu \mathrm{g} \mathrm{L}^{-1}$. The mean of characteristic masses derived from absorbance at each iron calibration standard for all calibrations was $13.3 \mathrm{pg}$ per $0.0044 \mathrm{~s}$ (SD $0.9 \mathrm{pg}$ per $0.0044 \mathrm{~s} n=16$ ), within $\pm 20 \%$ of the $12.0 \mathrm{pg}$ per $0.0044 \mathrm{~s}$ cited by the manufacturer. Initial and continuing calibration verification standards were analysed and were within $10 \%$ and $15 \%$ respectively of expected concentrations. A standard addition calibration was performed on one brain tissue digest. A $t$-test showed that the slope was not significantly different from that of the linear non-zero fit of the calibration performed in $1 \% \mathrm{HNO}_{3}$ at the $\alpha=0.05$ level.

\section{Statistical analysis}

Data were analysed using one-way ANOVA, having passed the Shapiro-Wilk test for normality, and this was followed by Dunnett's post-hoc analysis or Linear regression where appropriate (GraphPad Prism 4.0, San Diego, CA). In all cases, $P<0.05$ was taken as the level of significance. Linear Discriminant Analysis (Minitab) was used to assign the individual regional samples from each case into groups, based on data from multiple variables, and the proportion of samples correctly assigned to their true groups was reported for each parameter set considered.

\section{RESULTS}

Ferritin expression is selectively increased in MSA and unchanged in $P D$

In MSA basis pontis, there was an $\sim 2.5$ fold increase in ferritin protein as compared to control levels $(P<0.01 c f$ control), with no change apparent in PD tissue (Fig. 1a and d). A similar pattern was seen in the putamen where ferritin levels in MSA tissue were significantly increased by $\sim 4.5$ fold of control levels $(P<0.05 c f$ control, Fig. $1 \mathrm{c}$ and $\mathrm{d})$. In addition there 

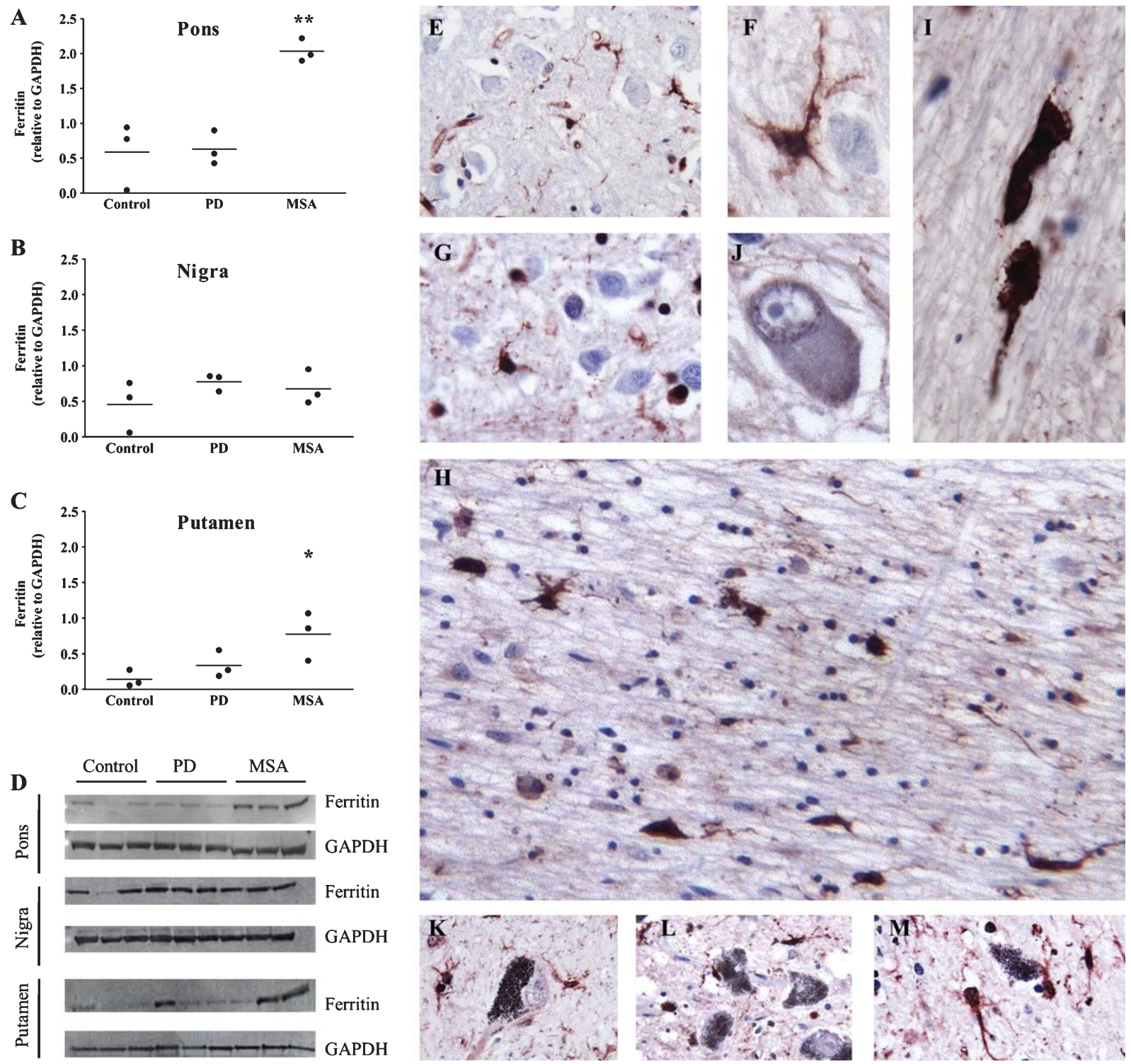

Fig. 1. Ferritin expression in control, PD and MSA brain. Ferritin signal relative to GAPDH was quantified by western blot in A) pons, B) substantia nigra and C) putamen. Individual values are shown, with the mean indicated by a black line. * represents significant difference from control levels, 1-way anova with Dunnett's post hoc, $\left({ }^{*} P<0.05,{ }^{* *} P<0.01\right)$. Representative blots for each region are shown D). Ferritin immunostaining in control E, F) PD G) and MSA H-J) pons. Ferritin immunostaining in control K) PD L) and MSA M) nigra. Scale bar represents $40 \mu \mathrm{m}$ in $\mathrm{E}, \mathrm{G}, \mathrm{H} \& \mathrm{~K}-\mathrm{M}$, and $10 \mu \mathrm{m}$ in $\mathrm{F}, \mathrm{J} \& \mathrm{I}$.

was an $\sim 1.4$ fold increase in ferritin in PD putamen $(P<0.05)$. In contrast, in the substantia nigra, ferritin levels remained unchanged in either PD or MSA as compared to control (both $P>0.05$, Fig. $1 \mathrm{~b}$ and d).

Immunohistochemical analyses of basis pontis sections from human control, PD and MSA tissue further supported differences in the intensity of ferritin expression. In control pons, ferritin was localised to microglia with no apparent neuronal staining (Fig. 1E), and the microglia displayed the typical features of a resting state with a round dense nucleus without a visible cytoplasm surrounding it and long thin processes (Fig. 1F). Similarly in PD basis pontis, ferritin was also predominantly expressed in microglia with an apparent resting morphology (Fig. 1G). In MSA pons the gross level of ferritin immunostaining appeared increased compared to both control and PD pons, confirming the observed increase by western blot (Fig 1H). Furthermore, in MSA basis pontis intense ferritin staining was seen in microglia which had thicker branches and an 
enlarged cytoplasm, a morphology associated with an activated state (Fig. 1I). There was no ferritin staining apparent in neurons in the pons of MSA cases (Fig. 1J). Immunohistochemical analysis of ferritin in the substantia nigra revealed the presence of microglia with an activated morphology clustered around the pigmented dopaminergic cells in both MSA and PD (Fig. 1K-M).

\section{Iron concentration, and ferritin-bound iron, is altered in MSA and PD}

In the basis pontis, total iron quantification with Graphite Furnace Atomic Absorption Spectroscopy (GFAAS) revealed an $\sim 1$ fold increase in iron in MSA as compared to control $(P<0.01)$, with levels in PD pons equivalent to control (Fig. 2a). The corresponding isothermal remanent magnetisation (IRM) measurements at temperatures of $5 \mathrm{~K}, 50 \mathrm{~K}$, and $150 \mathrm{~K}$ revealed an $\sim 1.6$ fold increase in IRM at $5 \mathrm{~K}$ in MSA pons as compared to control pons $(P<0.01)$, with PD IRM again being unchanged (Fig. 2c). The observed increased IRM signal in MSA pons at $5 \mathrm{~K}$ is consistent with MSA pons containing a higher concentration of nanoscale iron oxide particles in the tissue than either the control or PD pons, where indications are that these are ferritin cores.

In the substantia nigra, the pattern of iron and IRM in MSA and PD was reversed. Thus on average there was approximately a 2-fold increase in iron in PD as compared to control (although this did not reach significance in this small group, testing at $p<0.05$ ), with levels in MSA pons equivalent to control (Fig. 2b). The corresponding IRM signal from the same samples paralleled the trend indicated in the total iron data, revealing on average a 2-fold increase in IRM in PD nigra as compared to control nigra (approaching significance at $p=0.06$ ), with MSA IRM from the nigra again equivalent to control (Fig. 2d). The evidence for increased IRM signal in PD nigra is consistent with a higher concentration of iron being present in nanoparticulate form in the tissue than in either control or MSA substantia nigra. There are various forms of iron that may contribute to IRM signal at $5 \mathrm{~K}$, including ferritin iron oxide cores, and neuromelanin-bound iron, so it is not possible to differentiate the ferritin iron and neuromelanin iron fractions in the substantia nigra from these data alone [10]. However, lack of elevation in ferritin protein expression in the PD substantia nigra (Fig. 1B), and the increase in total iron and IRM in this same region, points towards an elevation of neuromelanin-bound iron (and a proportional, if not absolute, decrease in ferritin-bound iron) in the substantia nigra.

\section{Increased iron is stored within ferritin in MSA pons}

Linear regression analysis reveals a positive correlation between iron concentration and IRM at $5 \mathrm{~K}$ in tissue from both the pons $\left(r^{2}=0.95, P<0.0001\right)$ and substantia nigra $\left(r^{2}=0.97, P<0.0001\right)$ (Fig. $2 \mathrm{e}$ and $\mathrm{f}$ respectively). This indicates that the proportion of iron stored in the form of iron oxide clusters (as found in ferritin) increases in a linear fashion with total iron in the MSA pons (as opposed to an increase in the fraction of iron that is unbound or associated with low-molecular-weight species). There are no clear data in the literature showing accumulation of iron in the basis pontis, as most neuropathological data emphasise changes in the striatum in cases of MSA. Our own results show increased iron deposition in the pons in comparable density to observed changes in the striatum. A similar linear relationship between the IRM signal at $5 \mathrm{~K}$ and iron concentration in the substantia nigra is also observed.

\section{Free iron may be decreased in MSA pons}

Total tissue iron may be considered as that which is bound in ferritin, and that which is free or bound as individual ions to low molecular weight complexes such as transferrin (Tf). Notable exceptions include neuromelanin in the substantia nigra, which incorporates iron in ferritin-like oxyhydroxide clusters [15], and haemosiderin, reportedly a degradation product of ferritin, and which is more commonly observed in organs responsible for iron storage such as the liver. Tissue is primarily diamagnetic, and any magnetic signal from tissue at ultra-low temperatures, which is lost at higher temperatures, and follows ferritin-core-like magnetization saturation behaviour, would normally be attributable to the iron oxide cores in ferritin [13]. If we assume equivalent size distributions and biomineral properties of the ferritin cores in these pons tissue samples, then from inspection of the results shown in Fig. 2, it is evident that the proportion of non-ferritin-bound iron decreases in the MSA cases compared to controls, and to a greater extent than in PD. This indicates that in addition to an approximately 2-fold increase in iron concentration, the proportion of ferritin-bound iron also increases in the MSA pons. As shown previously, ferritin protein is elevated approximately 3-fold in this region of the brain (Fig. 1A). 
A

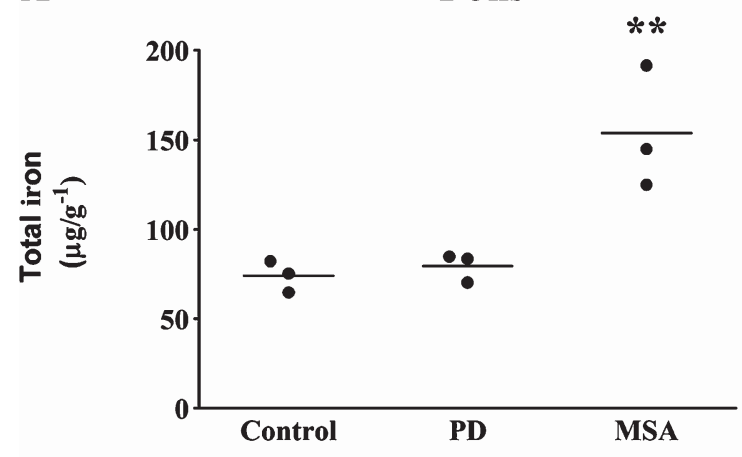

C

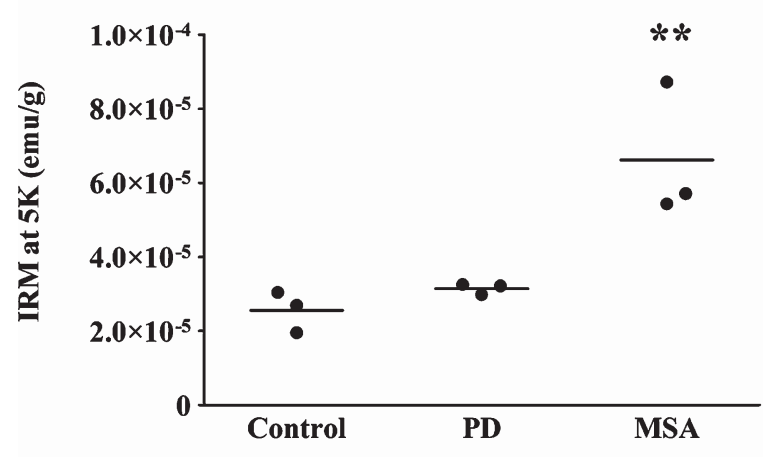

E

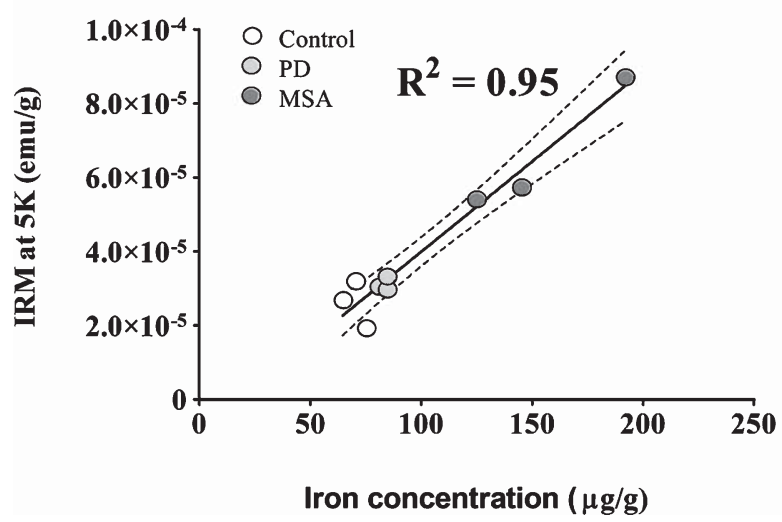

B

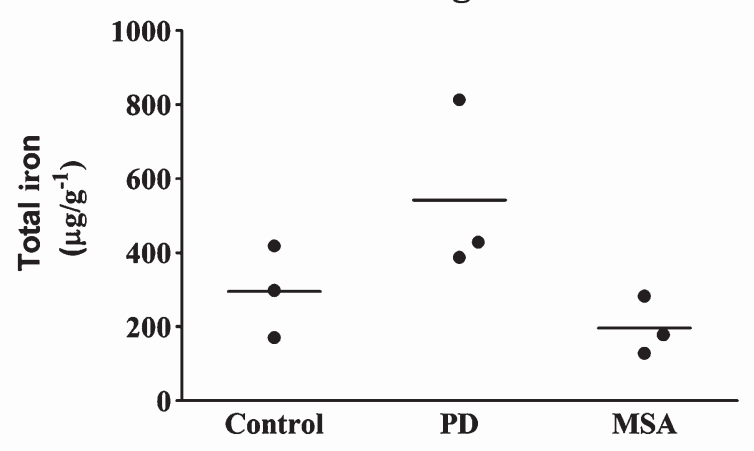

D

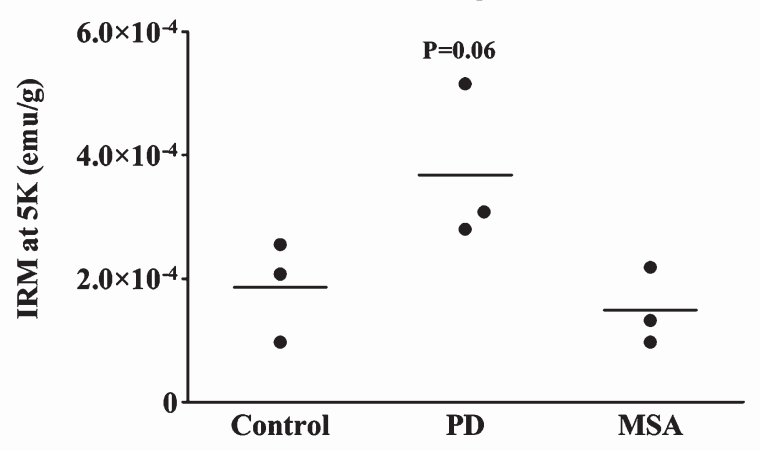

F

Nigra

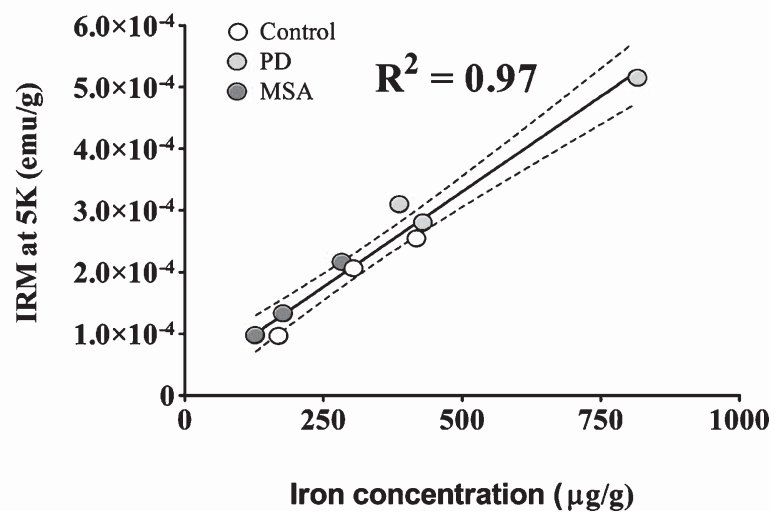

Fig. 2. Levels of iron and isothermal remanent megnetisation (IRM) in the pons and substantia nigra in control, PD and MSA brain. Total iron levels $\left(\mu \mathrm{g} / \mathrm{g}^{-1}\right)$ are shown in $\mathrm{A}$ ) pons and B) substantia nigra. IRM (emu/ $\mu \mathrm{g}^{-1}$ ) at 5 Kelvin in corresponding tissue is shown. Individual values are shown, with the mean indicated by a black line. * represents significant difference from control levels, 1-way anova with Dunnett's post hoc, $\left({ }^{* *} \mathrm{P}<0.01\right)$. IRM $\left(\mathrm{emu} / \mu \mathrm{g}^{-1}\right)$ at 5 Kelvin are plotted against total iron levels $\left(\mu \mathrm{g} / \mathrm{g}^{-1}\right)$ in E) pons and F) substantia nigra. $\mathrm{R}^{2}$ is shown. Line of best fit indicated by solid line, error by broken line.

Ferroportin and transferrin receptor expression is differentially altered in MSA and PD

In MSA pons, there was an $\sim 0.75$ fold decrease in ferroportin protein as compared to control levels $(P<0.01$ cf control), with no change apparent in PD tissue (Fig. 3A). A similar pattern was seen in the putamen where ferroportin levels in MSA tissue were decreased by $\sim 0.75$ fold of control levels $(P<0.05$ $c f$ control, Fig. 3C) with no significant change in PD putamen. In contrast, in the PD substantia nigra, there was an $\sim 1.4$ fold increase in ferroportin pro- 

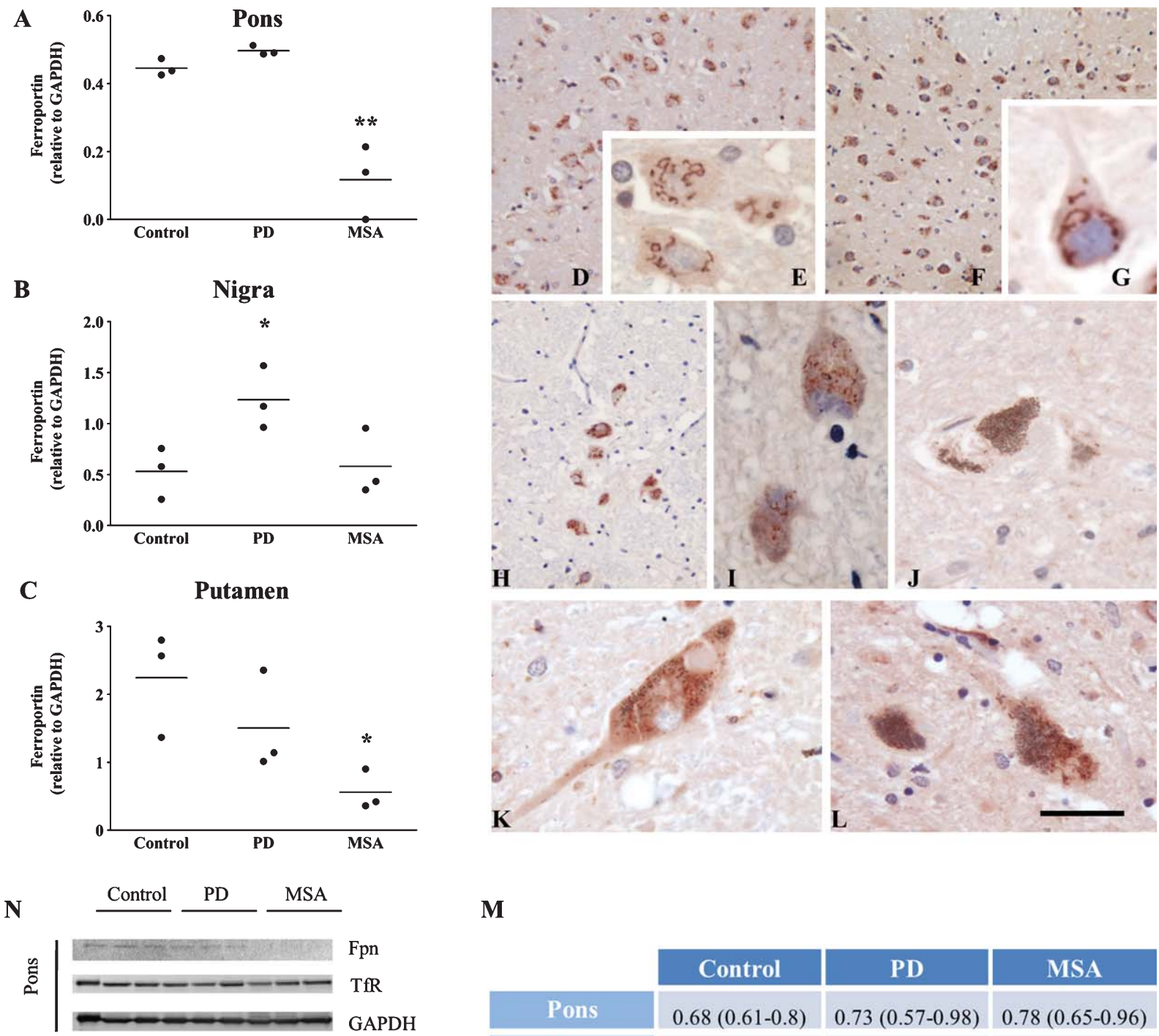

$\mathbf{M}$

\begin{tabular}{|c|c|c|c|}
\hline & Control & PD & MSA \\
\hline Pons & $0.68(0.61-0.8)$ & $0.73(0.57-0.98)$ & $0.78(0.65-0.96)$ \\
\hline Nigra & $4.25(3-5.2)$ & $4.75(3.8-5.7)$ & $3.69(3.3-4.4)$ \\
\hline Putamen & $1.53(1.0-1.8)$ & $0.68(0.6-0.8)$ & $0.91(0.7-1.2)$ \\
\hline
\end{tabular}
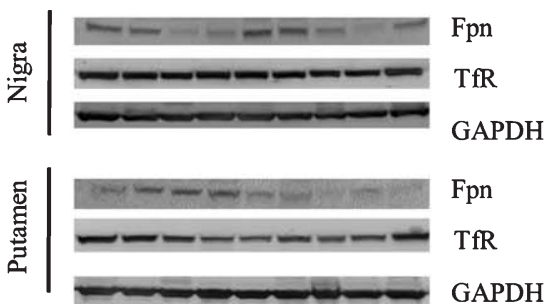

Fig. 3. Ferroportin (Fpn) and transferrin receptor (TfR) expression in control, PD and MSA brain. Ferroportin signal relative to GAPDH was quantified by western blot in A) pons, B) substantia nigra and C) putamen. Individual values are shown, with the mean indicated by a black line. *represents significant difference from control levels, 1 -way anova with Dunnett's post hoc, $\left({ }^{*} P<0.05,{ }^{* *} P<0.01\right)$. Ferroportin immunostaining in control D, E) PD F, G) and MSA FH, I pons and control J), PD K) and MSA L) substantia nigra neurons. Scale bar represents $200 \mu \mathrm{m}$ in D, F, H and $20 \mu \mathrm{m}$ in E, G, I-L. Transferrin receptor expression relative to GAPDH was quantified by western blot in pons, substantia nigra and putamen, mean and range are shown $\mathrm{M}){ }^{*}$ represents significant difference from control levels, 1 -way anova with Dunnett's post hoc, $\left({ }^{*} P<0.05\right)$. Representative blots for each brain region are shown $\mathrm{N}$ ).

tein as compared to control levels $(P<0.05 c f$ control $)$, with no change apparent in MSA substantia nigra (Fig. 3B).
Immunohistochemical analyses of pons sections from human control, PD and MSA tissue revealed a selective neuronal expression of ferroportin and a 

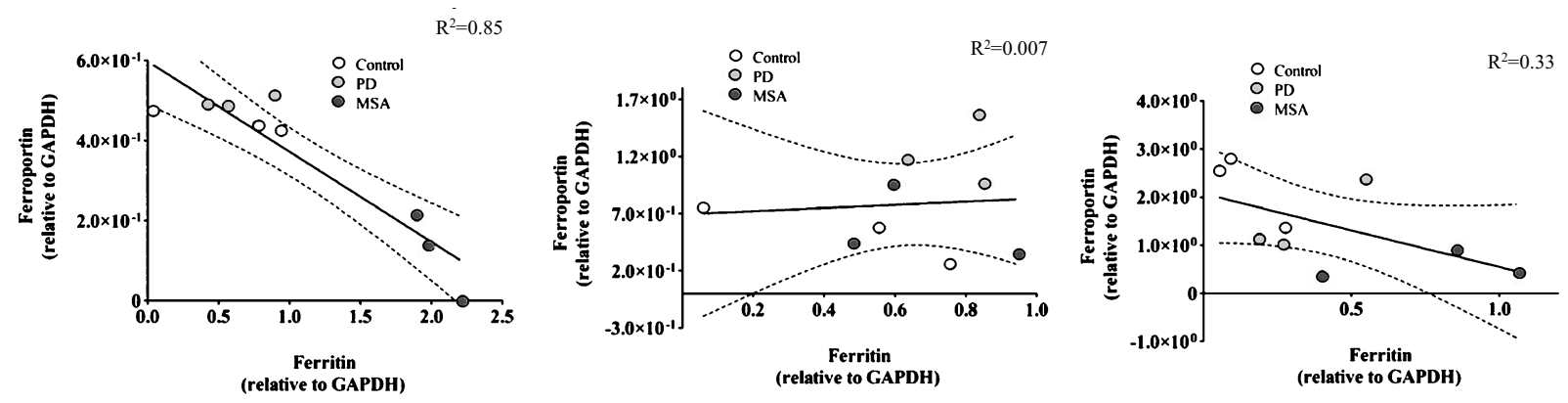

Fig. 4. Correlation between ferritin and ferroportin levels as measure by western blot in the pons, substantia nigra and putamen in control, PD and MSA brain. Ferritin and ferroportin signal relative to GAPDH was quantified by western blot in control, PD and MSA brain. Ferritin expression was plotted against ferroportin expression in A) pons, B) substantia nigra and c) putamen. $\mathrm{R}^{2}$ is shown. Line of best fit indicated by solid line, error by broken line.

co-localization with the Golgi apparatus. In control pons, ferroportin staining was apparent in most neurons displaying a filiform perinuclear distribution (Fig. 3D and 3E). In PD pons the pattern of ferroportin staining was similar to control with an intact filiform appearance (Fig. 3F, G). In MSA pons, the ferroportin signal appeared weaker and more diffuse (Fig. 3H, I). Analyses of sections from human control, PD and MSA substantia nigra similarly revealed a selective neuronal expression of ferroportin. Indeed the signal for ferroportin was prevalent in all pigmented neurons. However, in the PD substantia nigra expression of ferroportin appeared diffuse and fragmented in comparison to control and MSA tissues (Figs. 3J-L).

Transferrin receptor expression was unaffected in all groups in the substantia nigra and pons (Fig. 3M). However, in the PD putamen, transferrin receptor expression was significantly lower than in the control putamen $(P<0.05)$. This contrasts with MSA, where transferrin receptor expression was unchanged as compared to control putamen.

Linear regression analysis reveals a strong negative correlation between ferritin levels and ferroportin levels in the pons $\left(R^{2}=0.85, P<0.001\right)$ (Fig. $\left.4 \mathrm{a}\right)$. However, this strong negative correlation is not present in either the substantia nigra or putamen (Figs. $4 \mathrm{~b}$ and c).

\section{Microglia display activated morphology in MSA pons}

Immunohistochemical analysis of the microglial membrane specific protein ibal demonstrated that in MSA pons, microglia exhibit the morphology of an activated state: a large cell body with cytoplasmic hypertrophy and enlarged less defined nucleus and shorter thickened processes (Fig. 5c). This morphol- ogy was also apparent, albeit it to a lesser extent, in PD pons (Fig. 5b) but not in control pons (Fig. 5a). Positive pixel analysis of ibal stained tissue revealed an $\sim 10$ fold increase in iba1 positive pixels as compared to control levels $(P<0.01)$, and an $\sim 3.6$ fold increase in PD pons $(P<0.01 c f$ control) (Fig. 5d).

\section{Regional iron status differentiates MSA, PD, and healthy controls}

A summary of the quantitative differences in measures of iron status is provided in Table 1. Although the cases are carefully matched in terms of age and gender, the sample size ( $n=3$ in each disease group) means that some relationships already well-established in the literature (such as elevated iron in the PD SN compared to healthy controls) do not reach significance in the present study at the 5\% level using one-way ANOVA. Linear discriminant analysis (LDA) was used as a method appropriate to small groups to test whether individual samples are correctly assigned to their true disease groups on the basis of measured values of iron status in each brain region. As shown in Table 2a, the majority of samples were correctly assigned as controls, MSA, and PD when considered in terms of measured iron status values (ferritin, ferroportin, total iron, and IRM at $5 \mathrm{~K}$ ) for each region in turn. For basis pontis and putamen, 8 out of the 9 individual samples were correctly assigned in each region, and 7 out of 9 in the substantia nigra. Assignment of the PD samples to the PD disease group on the basis of iron status was $100 \%$ correct (9/9) in all three brain regions. The results in Table 2a indicate a high degree of discrimination between disease groups, supporting the hypothesis that modes of iron dysregulation are disease-specific. An even higher degree of success was achieved when considering each measure of iron status in turn, and 

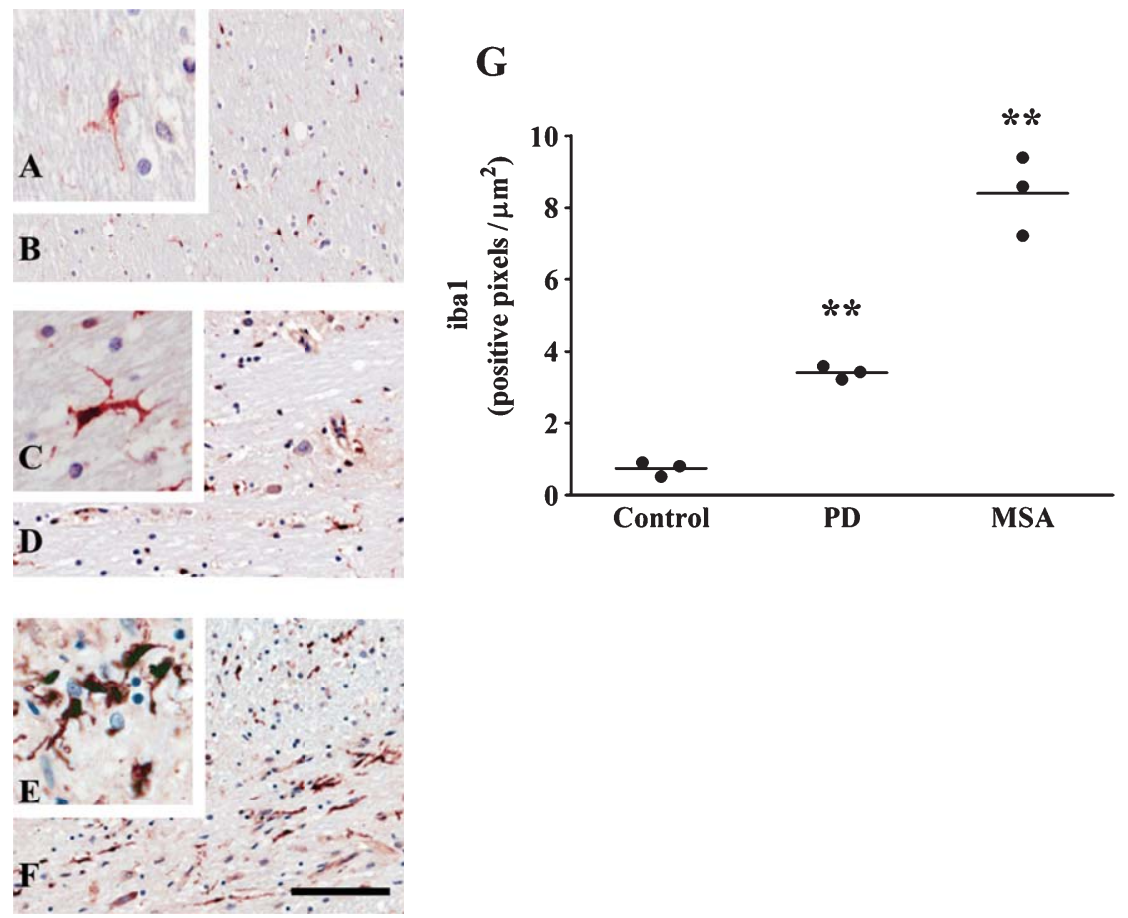

Fig. 5. Microglial morphology in control, PD and MSA pons. iba1 immunostaining in control A, B) PD C, D) and MSA E, F) pons. The number of iba1 positive pixels per $\mu \mathrm{m}^{2}$ was calculated G). Individual values are shown, with the mean indicated by a black line. * ${ }^{*}$ represents significant difference from control levels, 1-way anova with Dunnett's post hoc, $\left.{ }^{* *} P<0.01\right)$. Scale bar represents $30 \mu \mathrm{m}$ in A, C \& D and $75 \mu \mathrm{m}$ in B, D $\& \mathrm{E}$.

Table 1

Summary of differences between MSA, PD, and control (C) groups $(n=3)$, for measures of iron status. Using one-way analysis of variance (ANOVA), statistically significant increases $(\boldsymbol{\uparrow})$ and decreases $(\boldsymbol{\Downarrow})$ are indicated, with $\hat{\imath}$ and $\sqrt{ }$ used where values approach (but do not reach) significance at $P<0.05$

\begin{tabular}{|c|c|c|c|}
\hline & Basis pontis & Substantia nigra & Putamen \\
\hline Total iron concentration & $\begin{array}{l}\text { MSA } \uparrow \text { c.f. C, PD } \\
p<0.005\end{array}$ & $\begin{array}{l}\text { PD 仓 vs. MSA } \\
P=0.09\end{array}$ & Unchanged \\
\hline Particulate iron (IRM @ 5K) & $\begin{array}{l}\text { MSA } \uparrow \text { c.f. C, PD } \\
p<0.001\end{array}$ & $\begin{array}{l}\text { PD 仓े vs. MSA } \\
P=0.06\end{array}$ & $\begin{array}{l}\text { MSA } \uparrow \text { vs. C } \\
p<0.05\end{array}$ \\
\hline $\begin{array}{l}\text { Total vs. particulate iron } \\
\text { [concentration vs. IRM @ 5K] (all cases) }\end{array}$ & $\begin{array}{l}\text { Strong positive } \\
\text { correlation, }\left(r^{2}=0.95\right) \\
p<0.0001\end{array}$ & $\begin{array}{l}\text { Strong positive } \\
\text { correlation, }\left(r^{2}=0.96\right) \\
p<0.0001\end{array}$ & - \\
\hline Iron storage ferritin protein & $\begin{array}{l}\text { MSA } \uparrow \text { c.f. C, PD } \\
p<0.005\end{array}$ & $\begin{array}{l}\text { Unchanged } \\
p<0.005\end{array}$ & MSA \& PD $\boldsymbol{\uparrow}$ vs. C \\
\hline Iron export ferroportin protein & $\begin{array}{l}\text { MSA } \downarrow \text { c.f. C, PD } \\
p<0.005\end{array}$ & $\begin{array}{l}\text { PD } \uparrow \text { vs. } C \\
p<0.05\end{array}$ & $\begin{array}{l}\text { MSA } \Downarrow \text { vs. C, } \\
p<0.005\end{array}$ \\
\hline $\begin{array}{l}\text { Storage vs. export [ferritin vs. } \\
\text { ferroportin] (all cases) }\end{array}$ & $\begin{array}{l}\text { Strong negative } \\
\text { correlation, } p<0.001 \\
\left(r^{2}=0.85\right)\end{array}$ & $\begin{array}{l}\text { No correlation } \\
\left(r^{2}=0.01\right)\end{array}$ & $\begin{array}{l}\text { Weak negative } \\
\text { correlation } \\
\left(r^{2}=0.33\right)\end{array}$ \\
\hline Iron uptake transferrin protein & Unchanged & Unchanged & $\begin{array}{l}\text { PD } \downarrow \text { vs. C } \\
p<0.05\end{array}$ \\
\hline
\end{tabular}

assigning the individual samples on the basis of brain region (basis pontis, substantia nigra, putamen). These results are shown in Table $2 b$, where $100 \%$ of the samples were correctly assigned for ferritin, ferroportin, and IRM at $5 \mathrm{~K}$, and all but one (a control assigned as PD) were correctly assigned for total iron. This supports the hypothesis that there are region-specific differences in iron as a function of disease, in addition to the disease-specific modes of iron dysregulation evidenced in Table $2 \mathrm{a}$. 
Table $2 \mathrm{a}$

Linear Discriminant Analysis (LDA) of the individual cases, allocating each into disease groups (MSA, PD, control) on the basis of a) iron analyses performed for each region, and b) regional profile for a given analytical measure of iron. Correctly assigned samples are shown in bold font, incorrectly assigned are in brackets. a) LDA of the quantitative measures of iron status in each region, using ferritin, ferroportin, total iron, and particulate iron (IRM at $5 \mathrm{~K}$ ) as the variables, gives a high degree of discrimination between disease groups, supporting the hypothesis that modes of iron dysregulation are disease-specific

\begin{tabular}{llccc}
\hline & Allocated group & True group control & MSA & PD \\
\hline Basis pontis & Control & $\mathbf{2}$ & 0 & 0 \\
89\% success & MSA & 0 & $\mathbf{3}$ & 0 \\
& PD & $(1)$ & 0 & $\mathbf{3}$ \\
Substantia nigra & Control & $\mathbf{2}$ & $(1)$ & 0 \\
$78 \%$ success & MSA & $(1)$ & $\mathbf{2}$ & 0 \\
& PD & 0 & 0 & $\mathbf{3}$ \\
Putamen & Control & $\mathbf{2}$ & 0 & 0 \\
89\% success & MSA & 0 & $\mathbf{3}$ & 0 \\
& PD & $(1)$ & 0 & $\mathbf{3}$ \\
\hline
\end{tabular}

Table $2 b$

LDA of the samples by iron status, using the regions (basis pontis, substantia nigra, putamen) as variables, gives $100 \%$ success in assigning each sample to the correct disease group for three of the four independent measures of iron status. This supports the hypothesis that there are region-specific differences in iron as a function of disease. (Only 8 samples were included in this analysis; one matched PD SN sample was from another case.)

\begin{tabular}{llccc}
\hline & $\begin{array}{l}\text { Allocated } \\
\text { group }\end{array}$ & $\begin{array}{c}\text { True group } \\
\text { control }\end{array}$ & MSA & PD \\
\hline Ferritin & Control & $\mathbf{3}$ & 0 & 0 \\
$100 \%$ success & MSA & 0 & $\mathbf{3}$ & 0 \\
& PD & 0 & 0 & $\mathbf{2}$ \\
Ferroportin & Control & $\mathbf{3}$ & 0 & 0 \\
$100 \%$ success & MSA & 0 & $\mathbf{3}$ & 0 \\
& PD & 0 & 0 & $\mathbf{2}$ \\
Total iron & Control & $\mathbf{2}$ & 0 & 0 \\
$88 \%$ success & MSA & 0 & $\mathbf{3}$ & 0 \\
& PD & $(1)$ & 0 & $\mathbf{2}$ \\
Particulate iron (IRM @ 5K) & Control & 3 & 0 & 0 \\
$100 \%$ success & MSA & 0 & $\mathbf{3}$ & 0 \\
& PD & 0 & 0 & $\mathbf{2}$ \\
\hline
\end{tabular}

\section{DISCUSSION}

Conventional opinion holds that excess redox-active iron is implicated in neurodegeneration via production of reactive oxygen species which cause lipid peroxidation, protein damage and finally precipitate aggregation (reviewed in [16]). Although there is some controversy in the literature regarding alterations in the level of iron the substantia nigra in PD (reviewed in [17]), our demonstration of an approximately twofold increase in iron in PD substantia nigra compared to age-matched controls is consistent with similar increases demonstrated by several other groups using a range of techniques including X-ray fluorescence [18], spectrophotometry [19] and atomic absorption [20]. Furthermore, in a previous study of unfixed human tissue, using quantitative electron microprobe analysis, we demonstrated an approximately two-fold increase of cellular iron within individual nigral dopamine neurons in PD as compared to age-matched controls [21]. This approximate doubling of iron in the PD nigra, observed at cellular and whole tissue level, has thus been demonstrated to stand the test of time, and the demonstration of a strikingly similar level of increased iron by a range of techniques certainly adds validity to the GFAAS analysis employed in the present study. Here, we also demonstrate that the increased tissue iron and IRM signal is not accompanied by a statistically significant increase in the iron storage protein ferritin in the PD nigra, or a significant change in TfR expression. In isolation, the total tissue iron and ferritin expression data for the PD nigra support the findings of Dexter et al., who suggest that an decoupling of the relationship between iron and ferritin protein levels may lead to increased levels of redox active iron in the substantia nigra of PD [6]. We can extend this observation, as the IRM data, which parallel the increase in total tissue iron, indicate that the additional iron is coordinated in iron oxide clusters. Given the confirmed absence of ferritin elevation, this material that retains remanent magnetization at $5 \mathrm{~K}$ is most likely to be neuromelaninbound iron [10]. The elevated IRM signal in MSA pons tissue may reasonably be attributed to an elevated concentration of ferritin iron oxide cores, indicating that the excess iron in this tissue is ferritin-bound. This is the most straightforward interpretation of these data; it is acknowledged that the SQuID measurements performed in this study cannot unambiguously prove that the IRM signal in the pons is directly and proportionately due to ferritin concentration, as the presence of other iron biomineral phases within the ferritin, clustering of ferritin, or the presence of other iron nanoparticle inclusions in the tissue could bias the IRM value. Detailed analysis of the biomineral cores in ferritin extracted from these cases will be undertaken in future work.

Interestingly, we find the level of tissue iron, IRM and ferritin protein to be unchanged in the MSA nigra. While others have noted an increase in both tissue iron and ferritin in this region [6], our data are consistent with prior studies which found no evidence for a decoupling of ferritin expression from tissue iron concentration in MSA nigra; this feature appears specific to PD. The extent to which this feature is specific to the 
substantia nigra in PD remains to be confirmed. Other studies have shown an increase in iron in the putamen [22] and a decreased expression of transferrin receptors in the putamen in PD compared to age-matched controls [23]. We also note a decreased level of transferrin receptors in the putamen in $\mathrm{PD}$, suggestive of increased levels of free iron in this region. Furthermore, we see a trend towards increased ferritin expression in the putamen in PD. It is interesting that the decoupling between iron and ferritin reported in the PD nigra is not apparent in MSA despite the loss of nigral dopamine cells and similarities in nigral pathology between MSA and PD.

We contrast these observations in the substantia nigra with the relationship between iron and ferritin in the basis pontis, a region heavily affected by neurodegeneration in MSA but spared in PD and devoid of neuromelanin. As expected we found levels of tissue iron, IRM and ferritin to be statistically unchanged in PD pons as compared to control, but we found a twofold increase in total tissue iron concentration in the MSA pons mirroring the scale of the change observed by us and others in the PD nigra. Interestingly, in contrast to the PD nigra where ferritin expression was unchanged, we observed an approximately three-fold increase in the level of ferritin protein in the MSA pons. Thus, there is a distinct difference in the relationship between ferritin and total iron concentration in these two neurodegenerative diseases. We suggest that in MSA, although there is significantly elevated iron in the pons, this excess iron is sequestered by disproportionately elevated levels of the storage protein ferritin in reactive microglia, potentially leading to a net reduction in the levels of free iron. It remains unknown whether an initial increase in the number of activated microglia precedes an increase in ferritin, leading to an increased sequestration of iron, or an increased concentration of iron precipitates an increased production of ferritin and microglial activation. However, regardless of which is the initiating factor, the net result is a disproportionately high amount of ferritin protein (as determined by western blotting), as compared to the amount of iron, suggesting a deficit in bioavailable iron. As iron is vital to the function and survival of multiple cell types, we propose that a deficit in bioavailable iron may account for the involvement of multiple cell types in the pathology of MSA as compared to the more selective targeting of neurons in PD. As the presence of reactive microglia is well established in PD nigra, [24-27] the fact that we do not see a disproportionate increase in microglial ferritin in the PD nigra, as compared to in the MSA pons, is again indicative of a disease specific uncoupling of the relationship between ferritin and total iron concentration in MSA.

To further explore this hypothesis we investigated the neuronal iron export protein ferroportin. Iron homeostasis in the brain is performed by a variety proteins and a variety of cell types. Indeed, there are many mechanisms by which iron can be transferred between different cell types within the brain (reviewed in [28]). Given that synthesis of iron regulatory proteins is dependent on availability of iron for binding and that iron is readily transferred between cell types, it is important to examine relative levels of iron export and iron storage proteins at a system (whole tissue) level. Ferroportin expression typically parallels iron levels such that in times of iron excess more ferroportin is expressed (reviewed in [16]). As would be expected, we demonstrate a significant increase in ferroportin in the PD nigra as compared to control, with no change evident in MSA nigra, mirroring the changes we see in tissue iron concentration. The MSA pons is the exception, where despite a clear increase in tissue iron concentration, we observed a significant decrease in ferroportin expression. This decrease is accompanied by loss of the normal pattern of ferroportin distribution on the Golgi apparatus and implies possibly degradation of the iron exporter ferroportin and Golgi rearrangement [29]. This observation supports our hypothesis that the MSA pons may have a deficit in free cytosolic iron despite overall iron levels being increased, and that the cells' response to a perceived reduction in iron is a reduced expression of ferroportin. Consistent with previous reports, immunohistochemical analysis demonstrated the localisation of ferroportin to neurons in both the pons and substantia nigra, with some mild staining present in oligodendrocytes in the putamen [30]. There appeared to be two patterns of ferroportin subcellular signal. The most prevalent signal appeared to be bound to an intracellular cytoplasmic membrane and the other a more punctate signal suggestive of an endosomal localisation. Indeed it has been shown that ferroportin can be internalised and stored within endosomes prior to degradation [31]. Both patterns of expression were apparent in all positive neurons. Conventional opinion holds that ferroportin should be located on the extracellular membrane [28, 30]. Our findings, and those reported elsewhere, contradict this with an apparent lack of ferroportin signal on cell surface membranes [32]. Thus, although trafficking of ferroportin to the cell surface is likely regulated, this phenomenon certainly requires further investigation within the brain. 
Another avenue that warrants further study is the potential role of ceruloplasmin ( $\mathrm{Cp}$ ) in MSA. Cp plays a critical role in cellular iron efflux; it is responsible for oxidation of the ferrous iron exported by ferroportin, and impaired function of $\mathrm{Cp}$ impacts ferroportin stability, leading to reduced ferroxidase activity and mislocalization in the endoplasmic reticulum [33]. Mutant Cp has been shown in cell culture to drive loss of Golgi morphology, and degradation of ferroportin [29]. Hypoceruloplasminemia and mutant $\mathrm{Cp}$ have also been reported in cases of MSA [34, 35]; further investigation is required to determine the extent to which impaired Cp function might affect ferroportin in MSA.

The gene sequences for both ferritin and ferroportin contain an upstream $5^{\prime}$ iron response element (IRE) allowing translation to be controlled by the relative affinity of iron response protein 1 and 2 (IRP 1 and 2 respectively) themselves regulated by iron levels (reviewed in [16]). As the expression of both ferritin and ferroportin are controlled by this same mechanism, our observation that ferritin is increased, and ferroportin decreased, in MSA pons suggests that in MSA the levels of these two key homeostatic proteins are influenced by more than just iron levels. Others have shown that during liver regeneration, an increased activation of IRP also led to divergent effects on ferritin and transferrin receptor expression, in spite of both proteins having the same $5^{\prime}$ IRE [36]. We found the levels of IRP1 to be unchanged in MSA pons (data not shown). An alternative explanation for the apparently paradoxical alterations in ferritin and ferroportin in MSA pons involves the hormone hepcidin. Systemic iron homeostasis is maintained by the hepcidin which is secreted by hepatocytes in response to increased iron and signals for the internalisation and subsequent degradation of ferroportin (reviewed in [16]). Hepcidin has been identified throughout the brain in areas known to contain ferroportin and has also been shown to be induced by the pro-inflammatory cytokine interleukin6 (IL-6) [37, 38]. Interestingly, the presently observed alterations in levels of iron, ferritin and ferroportin are reminiscent of those underlying the anemia of chronic disease (ACD), a paradoxical condition mediated by pro-inflammatory cytokines whereby total body iron stores are elevated, but there is reduced bioavailable iron leading to an anemic condition [39]. We demonstrate that microglia in the MSA pons have a reactive profile with an enlarged irregular cell body and shorter thicker processes with little branching. Thus, we propose that a local inflammatory response, mediated by IL-6 secreted by activated microglia, may, via hepcidin, underlie the apparently paradoxical changes in ferroportin expression in MSA in the present study. Further, this situation may be more widespread than the pons, as there is much evidence of microglial activation throughout the MSA brain [40].

The link between iron levels and alpha synuclein is not well understood and any explanation would be speculative. However, recent evidence has demonstrated in vitro that alpha synuclein serves as a cellular ferrireductase, responsible for reducing iron (III) to bioavailable iron (II) [3]. As PD and MSA both involve aggregation of alpha synuclein, it is possible that the ferrireductase function of alpha synuclein is perturbed in these synucleiopathies. On the other hand, PD is associated with an increased expression of alpha synuclein which may lead to increased levels of bioavailable iron II. Indeed it is possible that both these mechanisms may contribute to different timepoints in the lengthy disease process. Accumulation of alpha synuclein, either as lewy bodies in PD or filamentous nuclear and cytoplasmic inclusions in neurons and glia in MSA, likely reflects an end-point stage reflective of stressed dying cells. The differences between the types of alpha synuclein aggregates in PD and MSA is poorly understood, but our findings suggest that Lewy bodies may form where there is excess iron and MSA aggregates where there are decreased levels of iron. The distribution of alpha synuclein pathology may be a reflection of differences in the regional susceptibility of some neurons and glia to changes in iron levels. Exploration of the specific role of alpha synuclein in iron dysregulation in all synucleinopathies clearly warrants further investigation.

In conclusion, while it is recognised that the low number of samples is a limitation of this study, our present findings raise the distinct possibility that despite an increase in total tissue iron concentration, there may a deficit in bioavailable iron in MSA. Future studies will determine the potential role of local inflammatory signalling in maintaining this paradoxical low bioavailable iron state. Furthermore, this deficit may account for the degeneration of multiple cell types in MSA pathology.

\section{ACKNOWLEDGMENTS}

We thank Prof J. Dobson and Dr M. Lees for advice concerning IRM measurement and analysis. NPV was supported by a Basic research fellowship from the Parkinson Society Canada. JFC acknowledges support for this study from EPSRC Postdoctoral Fellowship EP/D066654/1 and an RCUK Academic Fellowship. 
MEF was supported by a PhD studentship grant from the Alzheimer's Society, UK. AT acknowledges support from the Canadian Institutes of Health Research (MOP 84501) and the Parkinson Society of Canada. All tissue samples used in this study were provided from the Canadian Brain tissue Bank and were studied under ethical approval from the relevant REC (UK) and University Health Network Research Ethics Board. The GFAAS analysis was performed with support from EPSRC. The SQUID magnetometer used in this research was obtained through the Science City Advanced Materials project: Creating and Characterising Next Generation Advanced Materials project, with support from Advantage West Midlands (AWM) and part funded by the European Regional Development Fund (ERDF).

\section{REFERENCES}

[1] Forno LS (1996) Neuropathology of Parkinson's disease. J Neuropathol Exp Neurol, 55, 259-272.

[2] Crichton R \& Ward RJ (2008) Metal-based neurodegeneration - from molecular mechanisms to therapeutic strategies in Parkinson's disease. John Wiley \& Sons Ltd, 2006.

[3] Davies P, Moualla D \& Brown DR (2011) Alpha-synuclein is a cellular ferrireductase. PLoS One, 6, e15814.

[4] Ayton S, Lei P, Bush AI \& Finkelstein DI (2013) Alpha synuclein is a major regulator of iron homeostasis, in 11th Annual Conference on Alzheimer's and Parkinson's diseases (AD/PD). Florence, 2013.

[5] Riederer P, Dirr A, Goetz M, Sofic E, Jellinger K \& Youdim MB (1992) Distribution of iron in different brain regions and subcellular compartments in Parkinson's disease. Ann Neurol, 32 (Suppl) S101-s104.

[6] Dexter DT, Carayon A, Javoy-Agid F, Agid Y, Wells FR \& Daniel SE, et al. (1991) Alterations in the levels of iron, ferritin and other trace metals in Parkinson's disease and other neurodegenerative diseases affecting the basal ganglia. Brain, 114(Pt 4), 1953-1975.

[7] Dexter DT, Jenner P, Schapira AH \& Marsden CD (1992) Alterations in levels of iron, ferritin, and other trace metals in neurodegenerative diseases affecting the basal ganglia. The Royal Kings and Queens Parkinson's Disease Research Group. Ann Neurol, 32(Suppl), S94-S100.

[8] Friedman A, Arosio P, Finazzi D, Koziorowski D \& GalazkaFriedman J (2011) Ferritin as an important player in neurodegeneration. Parkinsonism Relat Disord, 17, 423-430.

[9] Arosio P \& Levi S (2010) Cytosolic and mitochondrial ferritins in the regulation of cellular iron homeostasis and oxidative damage. Biochim Biophys Acta, 1800, 783-792.

[10] Bolzoni F, Giraudo S, Lopiano L, Bergamasco B, Fasano M \& Crippa PR (2002) Magnetic investigations of human mesencephalic neuromelanin. Biochim Biophys Acta, 1586, 210-218.

[11] Hautot D, Pankhurst QA \& Dobson J (2005) Superconducting quantum interference device measurements of dilute magnetic materials in biological samples. Rev Sci Instrum, 76.

[12] Collingwood JF, Chong RK, Kasama T, Cervera-Gontard L, Dunin-Borkowski RE \& Perry G, et al. (2008) Threedimensional tomographic imaging and characterization of iron compounds within Alzheimer's plaque core material. $J$ Alzheimers Dis, 14, 235-245.

[13] Hautot D, Pankhurst QA \& Dobson J (2005) Superconducting quantum interference device measurements of dilute magnetic materials in biological samples. Rev Sci Instrum, 76.

[14] House E, Esiri M, Forster G, Ince PG \& Exley C (2012) Aluminium, iron and copper in human brain tissues donated to the Medical Research Council's Cognitive Function and Ageing Study. Metallomics, 4, 56-65.

[15] Zecca L, Gallorini M, Schunemann V, Trautwein AX, Gerlach M \& Riederer P, et al. (2001) Iron, neuromelanin and ferritin content in the substantia nigra of normal subjects at different ages: Consequences for iron storage and neurodegenerative processes. J Neurochem, 76, 1766-1773.

[16] Crichton RR, Dexter DT \& Ward RJ (2011) Brain iron metabolism and its perturbation in neurological diseases. $J$ Neural Transm, 118, 301-314.

[17] Friedman A, Galazka-Friedman J \& Koziorowski D (2009) Iron as a cause of Parkinson disease - a myth or a well established hypothesis? Parkinsonism Relat Disord, 15(Suppl 3), S212-S214.

[18] Earle KM (1968) Studies on Parkinson's disease including x-ray fluorescent spectroscopy of formalin fixed brain tissue. J Neuropathol Exp Neurol, 27, 1-14.

[19] Sofic E, Riederer P, Heinsen H, Beckmann H, Reynolds GP \& Hebenstreit G, et al. (1988) Increased iron (III) and total iron content in post mortem substantia nigra of parkinsonian brain. J Neural Transm, 74, 199-205.

[20] Griffiths PD \& Crossman AR (1993) Distribution of iron in the basal ganglia and neocortex in postmortem tissue in Parkinson's disease and Alzheimer's disease. Dementia, 4, 61-65.

[21] Oakley AE, Collingwood JF, Dobson J, Love G, Perrott HR \& Edwardson JA, et al. (2007) Individual dopaminergic neurons show raised iron levels in Parkinson disease. Neurology, 68, 1820-1825.

[22] De Volder AG, Francart J, Laterre C, Dooms G, Bol A \& Michel C et al. (1989) Decreased glucose utilization in the striatum and frontal lobe in probable striatonigral degeneration. Ann Neurol, 26, 239-247.

[23] Mash DC, Pablo J, Buck BE, Sanchez-Ramos J \& Weiner WJ (1991) Distribution and number of transferrin receptors in Parkinson's disease and in MPTP-treated mice. Exp Neurol, 114, 73-81.

[24] Block ML, Zecca L \& Hong JS (2007) Microglia-mediated neurotoxicity: Uncovering the molecular mechanisms. Nat Rev Neurosci, 8, 57-69.

[25] McGeer PL, Itagaki S, Boyes BE \& McGeer EG (1988) Reactive microglia are positive for HLA-DR in the substantia nigra of Parkinson's and Alzheimer's disease brains. Neurology, 38, 1285-1291.

[26] Langston JW, Forno LS, Tetrud J, Reeves AG, Kaplan JA \& Karluk D (1999) Evidence of active nerve cell degeneration in the substantia nigra of humans years after 1-methyl-4phenyl-1,2,3,6-tetrahydropyridine exposure. Ann Neurol, 46, 598-605.

[27] Imamura K, Hishikawa N, Sawada M, Nagatsu T, Yoshida M \& Hashizume Y (2003) Distribution of major histocompatibility complex class II-positive microglia and cytokine profile of Parkinson's disease brains. Acta Neuropathol, 106, 518-526.

[28] Moos T, Rosengren Nielsen T, Skjorringe T \& Morgan EH (2007) Iron trafficking inside the brain. J Neurochem, 103, 1730-1740.

[29] Persichini T, De Francesco G, Capone C, Cutone A, di Patti MC \& Colasanti M, et al. (2012) Reactive oxygen 
species are involved in ferroportin degradation induced by ceruloplasmin mutant Arg701Trp. Neurochem Int, 60, 360364.

[30] Moos T \& Rosengren Nielsen T (2006) Ferroportin in the postnatal rat brain: Implications for axonal transport and neuronal export of iron. Semin Pediatr Neurol, 13, 149-157.

[31] De Domenico I, Ward DM, Langelier C, Vaughn MB, Nemeth E \& Sundquist WI, et al. (2007) The molecular mechanism of hepcidin-mediated ferroportin down-regulation. Mol Biol Cell, 18, 2569-2578.

[32] Rouault TA (2001) Systemic iron metabolism: A review and implications for brain iron metabolism. Pediatr Neurol, 25, 130-137.

[33] Kono S, Yoshida K, Tomosugi N, Terada T, Hamaya Y \& Kanaoka S, et al. (2010) Biological effects of mutant ceruloplasmin on hepcidin-mediated internalization of ferroportin. Biochim Biophys Acta, 1802, 968-975.

[34] Yomono H, Kurisaki H, Murayama S, Hebisawa A, Miyajima H \& Takahashi Y (2003) An autopsy case of multiple system atrophy with a heteroallelic ceruloplasmin gene mutation. Rinsho Shinkeigaku, 43, 398-402.
[35] Kurisaki H, Yomono H, Murayama S \& Hebisawa A (2002) Multiple system atrophy with a-/hypo-ceruloplasminemia: Distribution of iron in brains of 2 autopsy cases. Rinsho Shinkeigaku, 42, 293-298.

[36] Cairo G, Tacchini L \& Pietrangelo A (1998) Lack of coordinate control of ferritin and transferrin receptor expression during rat liver regeneration. Hepatology, 28, 173-178.

[37] Zechel S, Huber-Wittmer K \& von Bohlen und Halbach O (2006) Distribution of the iron-regulating protein hepcidin in the murine central nervous system. J Neurosci Res, 84, 790-800.

[38] Nemeth E, Tuttle MS, Powelson J, Vaughn MB, Donovan A \& Ward DM, et al. (2004) Hepcidin regulates cellular iron efflux by binding to ferroportin and inducing its internalization. Science, 306, 2090-2093.

[39] Weiss G \& Goodnough LT (2005) Anemia of chronic disease. N Engl J Med, 352, 1011-1023.

[40] Ishizawa K, Komori T, Sasaki S, Arai N, Mizutani T \& Hirose $\mathrm{T}$ (2004) Microglial activation parallels system degeneration in multiple system atrophy. J Neuropathol Exp Neurol, 63, $43-52$ 